\title{
Lurasidone Induced Mania in a Case of Treatment Resistant Schizophrenia: A Case Report
}

\author{
Ashok Kumar* \\ Assistant Professor, Department of Psychiatry, S.N. Medical College, Jodhpur (Rajasthan), India \\ *Corresponding Author: Ashok Kumar, Assistant Professor, Department of Psychiatry, S.N. Medical College, Jodhpur (Rajasthan), India.
}

Received: September 09, 2019; Published: October 15, 2019

DOI: 10.31080/ASNE.2019.02.0115

\begin{abstract}
Antidepressant induced manic episode is well-established phenomenon in psychiatric literature. Induction of mania after use of antipsychotic medications is little known entity. We report a case of lurasidone induced mania in a case of treatment resistant schizophrenia which resolved within two weeks of lurasidone discontinuation.
\end{abstract}

Keywords: Treatment Resistant Schizophrenia; Lurasidone

Mr. BR a 26-year young male presented for the treatment of schizophrenia. Earlier he received four second generation and three of first generation antipsychotics with optimum dose and duration but with little or no response. In October 2016 he was finally started on clozapine with final diagnosis of resistant schizophrenia [1]. Over the six weeks of follow up his PANSS [2] score dropped from 88 to 59 and was maintained over the next four months. In March 2017, during routine blood count monitoring neutropenia was discovered resulting in discontinuation of clozapine and relapse of schizophrenia within a week. Considering the resistant status of his illness he was admitted in psychiatric unit and ECT was started as next line of treatment. Patient showed little response at the end of three weeks of ECT (7 sessions) and it was decided to reintroduce clozapine with daily monitoring of complete blood count. On third day of clozapine $(25 \mathrm{mg}$ ) the complete blood count dropped to 2700 (Absolute neutrophil count 900) from day one level of 6500 (Absolute neutrophil count 2200). Clozapine was dropped again temporarily before taking another re challenge with lithium cover [3]. This particular attempt was dropped as patient suffered with dramatic decrease in absolute neutrophil count within two days period.
Since patient was resistant to both typical as well as atypical antipsychotics and failed to respond with electroconvulsive therapy it was decided to start Lurasidone which was never used in this particular patient. Lithium (1200 mg per day) was continued as patient remained extremely aggressive during this period. At $10^{\text {th }}$ day of follow up patient showed some improvement in aggressive behavior while showing no response to delusions and hallucinations. Similarly no limiting side effects of lurasidone were reported and dose was increased to $80 \mathrm{mg}$ per day. On $20^{\text {th }}$ day of lurasidone, patient presented with increased talkativeness, decreased need for sleep, distractibility, intention to marry the most beautiful girl, irritability, stubbornness and increased physical activity. It was presumed to be a case of lurasidone induced mania and lurasidone was discontinued. On next follow up (10 June 2017) patient showed significant decrease in manic symptoms. His YMRS [4] score dropped from 18 to 7 within two-weeks of stopping lurasidone. Later on patient was stabilized on combination of typical (Flupenthixol decanoate) and atypical (olanzapine) antipsychotics and remains under control till date, albeit with limited success. No affective symptoms reported over the last two years of follow up. On retrospective chart review it was confirmed that patient never showed affective symptoms since 2010 , the time when he was first diagnosed with schizophrenia. 


\section{Discussion}

This is a probable case of Lurasidone induced mania. The presumptive association with lurasidone appears confirmed as the manic symptoms were resolved on discontinuation of lurasidone without any additional treatment and patient reported no significant affective symptoms in last two years after discontinuation of lurasidone. The criteria for diagnosis of mania are met in this patient as patient had elevated mood, decreased need for sleep, increased physical activity, grandiosity, distractibility and talkativeness [5].

We considered the possibility of schizoaffective disorder which can present with concomitant symptoms of schizophrenia and mood disorder. However on retrospective chart review it was found that the required affective symptoms to diagnose schizoaffective disorder never occurred in the past 10 years of illness. Patient had continuous illness over the last 10 years and no complete remission occurred over the course of illness. In addition disappearance of mood symptoms on discontinuation of lurasidone points toward lurasidone induced mood episode.

Affective symptoms are common occurrence in individuals with schizophrenia and reported in $30 \%$ of individuals with diagnosis of schizophrenia. However the above-described patient never had any noticeable affective symptoms over the course of illness. Disappearance of affective symptoms after discontinuation of lurasidone almost rules out the possibility of observed manic episode as part of illness.

Although the exact etiology of lurasidone induced manic episode is not known but it is possible that it acts in similar fashion alike other antidepressants [6]. The manic symptoms occurred 10 days after the start of lurasidone, a known dopamine antagonist. To our knowledge this is the first case of lurasidone induced mania in a patient with resistant schizophrenia. Doctors considering option of lurasidone in schizophrenia and bipolar depression should be cautious of precipitation of manic episode. This is highly relevant as episode occurred despite ongoing treatment with mood stabilizer.

\section{Bibliography}

1. Meltzer HY. "Treatment-resistant schizophrenia-the role of clozapine". Current medical Research and Opinion 14 (1997): $1-20$.

2. Kay SR., et al. "The positive and negative syndrome scale (PANSS) for schizophrenia". Schizophrenia Bulletin 13 (1987): 261-276.

3. Aydin M., et al. "Continuing clozapine treatment with lithium in schizophrenic patients with neutropenia or leukopenia: brief review of literature with case reports". Therapeutic advances in psychopharmacology 6 (2016): 33-38.

4. Young RC., et al. "A rating scale for mania: reliability, validity and sensitivity". The British Journal of Psychiatry 133 (1978): 429-435.

5. American Psychiatric Association. Diagnostic and statistical manual of mental disorders (DSM-5®). American Psychiatric Pub (2013).

6. Dilsaver SC and Greden JF. "Antidepressant withdrawal-induced activation (hypomania and mania): mechanism and theoretical significance". Brain Research Reviews 7 (1987): 2948.

\section{Volume 2 Issue 11 November 2019 \\ (C) All rights are reserved by Ashok Kumar.}

